\title{
Uric Acid Level is Positively Associated With NT-proBNP Concentration in Slovak Heart Failure Patients
}

\author{
P. SABAKA ${ }^{1}$, A. DUKAT ${ }^{2}$, J. GAJDOSIK ${ }^{3}$, M. CAPRNDA ${ }^{4}$, M. BENDZALA $^{1}$, F. SIMKO $^{5,6,7}$ \\ ${ }^{1}$ Department of Infectology and Geographical Medicine, Faculty of Medicine, Comenius \\ University, Bratislava, Slovakia, ${ }^{2}$ Fifth Department of Internal medicine, Faculty of Medicine, \\ Comenius University, Bratislava, Slovakia, ${ }^{3}$ Slovak Medical Chamber, Bratislava, Slovakia, ${ }^{4}$ First \\ Department of Internal medicine, Faculty of Medicine, Comenius University in Bratislava, \\ Bratislava, Slovakia, ${ }^{5}$ Institute of Pathophysiology, Faculty of Medicine, Comenius University, \\ Bratislava, Slovakia, ${ }^{6}$ Third Department of Internal Medicine, Faculty of Medicine, Comenius \\ University, Bratislava, Slovakia, ${ }^{7}$ Institute of Experimental Endocrinology, Biomedical Research \\ Center, Slovak Academy of Sciences, Bratislava, Slovakia
}

Received April 12, 2019

Accepted May 23, 2019

Epub Ahead of Print August 23, 2019

\section{Summary}

Increased concentration of uric acid (UA) is positively associated with the clinical severity but negatively associated with the prognosis of heart failure (HF). However, data related to the association between UA concentration and $\mathrm{N}$-terminal pro brain natriuretic peptide (NT-proBNP) are still lacking. The aim of the study was to analyze the relationships between UA, NT-proBNP, clearance of creatinine and NYHA function class and echocardiographic variables in the Slovak population of primary care patients diagnosed with HF. The association between UA and NT-proBNP was assessed by multivariate analysis. 848 patients (402 men, 446 women) with HF were included in the study. NT-proBNP correlated with UA in both men and women after adjustment based on age, BMI and glomerular filtration rate $(r=0.263, p<0.0001 ; r=0.293, p<0.0001)$. UA concentration rose with the severity of the NYHA class and was significantly higher in patients with moderate and severe systolic dysfunctions as well as with diastolic dysfunction in the multivariate analysis. In conclusion, our study in Slovak population with HF has revealed a positive correlation between the concentration of UA and NT-proBNP, and the independency of this association on confounding factors. The results support the role of UA as a biochemical marker of HF severity and prognosis.

\section{Key words}

Uric acid • Heart failure • NT-proBNP • Confounding factors • Biochemical marker

\section{Corresponding author}

F. Simko, Institute of Pathophysiology, Faculty of Medicine, Comenius University, Sasinkova 4, 81108 Bratislava, Slovakia. Fax: +421-(0)2-59357601. E-mail: fedor.simko@fmed.uniba.sk

\section{Introduction}

Heart failure (HF) represents a major public health problem. Its prevalence is rapidly growing, and it is estimated that more than 20 million individuals suffer from heart failure worldwide (Benjamin et al. 2017). Despite improvements in HF therapy, its prognosis remains poor (Bui et al. 2011, Ruliope et al. 2010, Simko and Simko 1999, Simko et al. 2003).

Therefore, research which is focused on finding new potential targets in the treatment of HF, is of the highest priority and heart remodeling and failure continuously attracts the attention of clinical and experimental cardiology (Lacko et al. 2018, Cundrle et al. 2018, Simko and Pechanova 2010, Simko et al. 2018a, Simko et al. 2018b).

Uric acid (UA), a by-product of purine 
metabolism, is of great significance in modern preventive cardiology, because of its apparent role in the pathogenesis of cardiovascular diseases. The first evidence associating hyperuricemia with increased cardiovascular risk emerged in 1951, when Gertler et al. (1951) postulated the existence of a connection between hyperuricemia and coronary heart disease. Since then, the potential role of UA in various cardiovascular pathologies has emerged in numerous epidemiologic studies (Cibicková et al. 2017, Fang et al. 2000, Timóteo et al. 2013). There is a substantial body of evidence indicating that hyperuricemia might participate in the pathogenesis of HF. The enhancement of UA concentration increases the risk of the development of $\mathrm{HF}$ and even worsens HF prognosis (Huang et al. 2014, Hare et al. 2003). Indeed, a large meta-analysis involving 1,456 patients has revealed the predictive role of UA in all-cause mortality in heart failure (Tamariz et al. 2011).

The pathophysiologic background of the relation between increased UA concentration and the development and progression of HF has not been fully elucidated yet. However, a hypothesis that excessive formation of reactive oxygen species (ROS) plays a pivotal role in this association has been proposed. UA is produced in the pathway resulting also in the formation of ROS (Sougstad et al. 1996) and it is believed that ROS plays an important role in the pathogenesis of HF (Wang et al. 2018, Liu et al. 2018). High UA concentration may therefore indicate the excessive ROS formation (McCord et al. 1985). In the pathogenesis of HF, enhanced ROS production participates in a mechanoenergetic uncoupling of the myocardium, which results in a decrease of contractility despite normal or increased energetic consumption (Hare et al. 2003).

B-type natriuretic peptide or brain natriuretic peptide (BNP) is produced in the left atrium and ventricle; it stimulates natriuresis and inhibits the effects of renin-angiotensin-aldosterone system. N-terminal B-type pro BNP is a well-established diagnostic and prognostic marker of HF (Hill et al. 2014, Kim et al. 2011). Its concentration reflects the severity of HF and correlates with clinical variables, such as New York Heart Association (NYHA) class and 6-min walk distance (Williams et al. 2005, Frantz et al. 2012). Despite the known associations between UA concentration and the risk of HF development, severity and outcome, there is no reliable evidence that UA concentration independently correlates with HF severity as diagnosed by a well established laboratory biomarker, such as NT-proBNP.
The potential evidence of a correlation between UA and NT-proBNP may confirm the importance of UA concentration as an additional biomarker of HF severity. Therefore, we aim to show whether or not there is a correlation between UA and NT-proBNP in patients with chronic HF among Slovak outpatient population.

\section{Methods}

\section{Patients}

We conducted a multicentric cross sectional study focused on the assessment of clinical features, comorbidities and therapy of patients with chronic heart failure. Consecutive patients were recruited by cardiologists on an outpatient basis. Heart failure was diagnosed according to the European Society of Cardiology Guidelines for the diagnosis and treatment of acute and chronic heart failure (Ponikowski et al. 2016). Patients were considered to suffer from HF if they exhibited typical symptoms (dyspnoea, fatigue, ankle swelling), signs (elevated jugular venous pressure, pulmonary crackles and peripheral oedema), NT-proBNP $>125 \mathrm{pg} / \mathrm{ml}$ and one of two additional criteria (demonstrated relevant structural heart disease and/or diastolic dysfunction) or if they showed signs and symptoms as well as a left ventricle ejection fraction (LVEF) less than $40 \%$ by echocardiography. Patients were stratified according to the systolic function impairment defined by echocardiographically determined LVEF according to European Society of Cardiology Guidelines for the diagnosis and treatment of acute and chronic heart failure (Ponikowski et al. 2016). Patients with $\mathrm{LVEF} \geq 50 \%$ were classified as $\mathrm{HF}$ with preserved LVEF (HFpEF), those with LVEF between $49-40 \%$ as HF with mid-range LVEF (HFmrEF) and those with LVEF below $40 \%$ as HF with reduced EF (HFrEF). VLEF was measured by echocardiography using he modified biplane Simpson's rule.

Patients older than 18 years were recruited proportionally from all regions of Slovakia. The exclusion criteria included a history of oncologic disease except total remission, pregnancy, thyrotoxicosis, end stage kidney disease and therapy by allopurinol. These information were obtained using a questionnaire. All patients signed an informed consent.

\section{Declarations}

Ethics approval and consent to participate: 
Written informed consent was obtained from all the participants before recruitment. The protocol was approved by the Ethical Committee of the Ministry of Health of Slovak Republic, and the study was conducted according to the Declaration of Helsinki.

\section{Blood sampling and processing}

At the time of the recruitment, blood from the cubital vein had been sampled after at least $12 \mathrm{~h}$ of fasting using Vacutainer closed system for serum sampling. The samples remained for $30 \mathrm{~min}$ at room temperature and subsequently, the supernatant was separated by centrifugation for $10 \mathrm{~min}$ at $3000 \mathrm{rpm}$. The supernatant was used for the analysis of serum NT-proBNP, creatinine and UA. Creatinine clearance (CCR) was calculated using CKD-EPI formula.

\section{Laboratory assays}

Creatinine was measured by modified enzymatic method (Dialab, Wr. Neudorf, Austria); UA was measured by an enzymatic method (Erba Lachema, Brno, Czech Republic); clearance of creatinine was calculated using MDRD formula; NT-proBNP plasmatic concentration was determined by chemiluminescence (Cobas, Roche, Basel, Switzerland).

\section{Statistics}

The data are provided as median and $25^{\text {th }}$ and $75^{\text {th }}$ percentile. Normal distribution of quantitative variables was verified using Kolmogorov-Smirnov test. The differences between quantitative variables were compared using the $t$-test for variables with normal distribution and Mann-Whitney test for variables without normal distribution. The associations between NT-proBNP, creatinine and UA were assessed using Pearson's (if normal distribution) and Spearman's (if not normal distribution) correlation coefficient. To exclude the effects of confounding factors, partial Spearman correlation was later used. To determine if UA is an independent predictor of NT-proBNP, multiple linear regression, which included other independent variables (clearance of creatinine, BMI, losartan use, gender and age), was used. To compare the UA concentration between various groups, multivariate analysis of variance (MANOVA) with post hoc Tukey's test was used. Values of $\mathrm{p}<0.05$ were considered as statistically significant. For statistical analysis, SPSS version 20 for Windows was used.

\section{Results}

\section{Patient characteristics}

Eight hundred and forty-eight (402 men, 446 women) consecutive patients were included in the study according to the inclusion and exclusion criteria and had complete set of biochemical, clinical and echocardiographic variables for analysis; $5 \%$ of them were in NYHA I, $51 \%$ were in NYHA II, $40 \%$ were in NYHA III and $4 \%$ were in NYHA IV. $26 \%$ of patients had HFpEF, $43 \%$ had HFmrEF and $31 \%$ had HFrEF. Age, BMI, CCR, NT-proBNP and uric acid are presented in Table 1 .

\section{Correlation between NT-proBNP and other variables}

NT-proBNP correlated positively with uric acid concentration in both men and women. To exclude the effects of confounding factors (age, CCR, gender, BMI, losartan therapy) on the association between NT-proBNP and UA, partial Spearman linear regression was used. After excluding the effects of confounding factors, CCR and BMI correlated with NT-proBNP only in men (Table 2).

Table 1. Patients' characteristics.

\begin{tabular}{lccccc}
\hline & Median & $\begin{array}{c}\text { Men } \mathbf{4 0 2} \\
\text { Interquartile range }\end{array}$ & Median & $\begin{array}{c}\text { Women N=446 } \\
\text { Interquartile range }\end{array}$ & p \\
\hline Age & 70 & $61-78$ & 75 & $67-81$ & $<0.0001$ \\
CCR $(\mathrm{ml} / \mathrm{min})$ & 71.3 & $58.9-87.3$ & 59.6 & $49.0-73.9$ & $<0.0001$ \\
Uric acid $($ Hmol/l) & 383.0 & $318.8-440.3$ & 336.0 & $285.0-403.0$ & $<0.0001$ \\
NTproBNP $(\mathrm{pmol} / \mathrm{l})$ & 658.5 & $169.8-1701.0$ & 430.0 & $156.8-1042.0$ & $<0.0001$ \\
BMI & 28.7 & $25.9-31.6$ & 30.04 & $26.2-34.4$ & $<0.001$ \\
\hline
\end{tabular}

BMI - body mass index, CCR - clearance of creatinine, $\mathrm{p}$ - probability, UA - uric acid. 
Table 2. Partial Spearman correlation between NT-proBNP and other variables.

\begin{tabular}{lcccc}
\hline & \multicolumn{2}{c}{ Men $\mathbf{N = 4 0 2}$} & \multicolumn{2}{c}{ Women $\mathbf{N}=\mathbf{4 4 6}$} \\
& Spearman $\mathbf{~}$ & $\mathbf{p}$ & Spearman $\mathbf{p}$ & $\mathbf{p}$ \\
\hline$A$ & 0.263 & $<0.0001^{\mathbf{a}}$ & 0.293 & $<0.0001^{\mathbf{a}}$ \\
$C C R$ & -0.035 & $<0.05^{\mathbf{b}}$ & -0.018 & $0.282^{\mathbf{b}}$ \\
$B M I$ & -0.064 & $<0.05^{\mathbf{c}}$ & -0.015 & $0.350^{\mathbf{c}}$ \\
\hline
\end{tabular}

a adjusted based on confounding factors: age, CCR, BMI, losartan therapy, ${ }^{\mathbf{b}}$ adjusted based on confounding factors: age, UA, BMI, losartan therapy, ' adjusted based on confounding factors: age, UA, CCR, losartan therapy, BMI - body mass index, CCR - clearance of creatinine, $\mathrm{p}$ - probability, UA - uric acid.

\section{Multiple linear regression model}

In the multiple linear regression model, NT-proBNP was independently predicted by uric acid concentration, age, gender, BMI, losartan therapy and CCR. The coefficient of determination for this model was $\mathrm{R}^{2}=0.177$ and $\mathrm{p}<0.0001$. Linear regression of NT-pro $\mathrm{BNP}$ and uric acid concentration is shown in Figure 1.

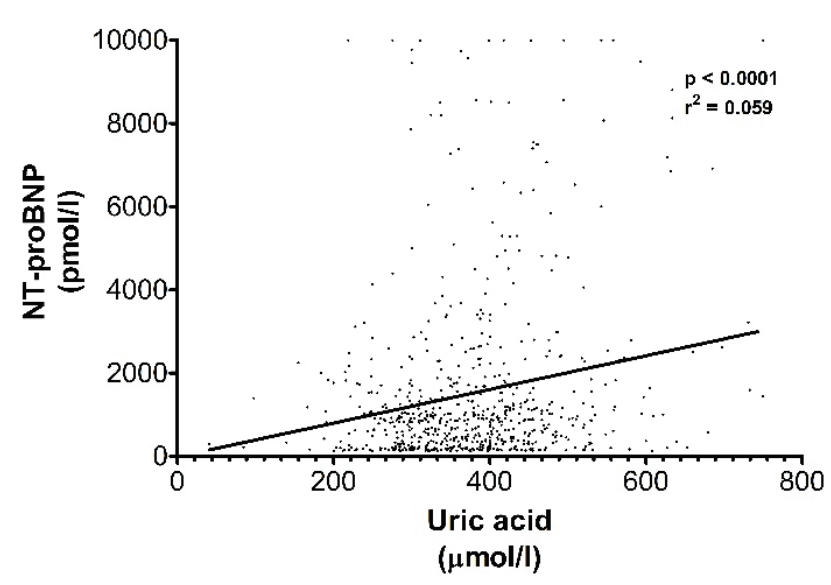

Fig. 1. Linear regression of uric acid concentration $(\mu \mathrm{mol} / \mathrm{l})$ and NT-pro BNP (pmol/l).
$U A$ concentration among different HF severity groups

After adjustment based on age, BMI and CCR, different NYHA groups differed in UA concentration, with the highest UA level in NYHA IV and the lowest in NYHA I (Table 3). Similarly, groups discriminated by left ventricular ejection fraction (LVEF) differed in UA concentration. The HFrEF group had higher UA concentrations than the HFpEF group and the HFmrEF group. There was not a statistically significant difference in the concentration of UA between HFpEF and HFmrEF groups (Table 3).

\section{Discussion}

Our cross sectional multicentric study in Slovak population with $\mathrm{HF}$ has revealed that the plasmatic concentration of UA positively correlates with NT-proBNP, the well-established marker of HF severity and prognosis, and also, that this association is independent of factors that are possibly confounding. Decisive evidence supporting this association was up to this time lacking. Moreover, the higher level of uricemia was associated with more severe HF.

Table 3. Uric acid concentration in different groups by clinical and echocardiographic variables.

\begin{tabular}{|c|c|c|c|c|c|}
\hline NYHA class & NYHA I & NYHA II & NYHA III & NYHA IV & p (MANOVA) \\
\hline$U A(\mu \mathrm{mol} / \mathrm{l})$ & $\begin{array}{c}293.5^{*} \\
(264.5-404.3)\end{array}$ & $\begin{array}{c}356.0 * \\
(299.0-426.0)\end{array}$ & $\begin{array}{c}383.0^{*} \\
(318.0-444.8)\end{array}$ & $\begin{array}{c}397.0 * \\
(351.5-444.0)\end{array}$ & $<0.0001$ \\
\hline \multicolumn{6}{|c|}{ * median differs from all other groups by post hoc test } \\
\hline Systolic function & HFpEF & $\mathbf{H F}$ & & HrEF & p (MANOVA) \\
\hline$U A(\mu \mathrm{mol} / \mathrm{l})$ & $\begin{array}{c}341.0^{\ddagger} \\
(284.5-402.5)\end{array}$ & $\begin{array}{r}37 \\
(301 .\end{array}$ & & $\begin{array}{c}391.0 \\
(328.5-442.5)\end{array}$ & $<0.0001$ \\
\hline
\end{tabular}

\footnotetext{
${ }^{\ddagger}$ groups HFpEF and HFmrEF differ significantly from the group HFrEF. There was no significant difference between groups HFpEF and HFmrEF

Variables are determined as median and interquartile range, adjusted based on age, body mass index, gender, creatinine clearance. HFpEF - heart failure with preserved ejection fraction, $\mathrm{HFmrEF}$ - heart failure with mid-range ejection fraction, $\mathrm{HFrEF}$ - heart failure with reduced ejection fraction, NYHA - New York Heart Association, p - probability, MANOVA - multivariate analysis of variance, UA - uric acid.
} 
UA and NT-proBNP: Plasmatic NT-proBNP concentration is a well-established biomarker of HF severity and highly correlates with the clinical severity of HF (Hill et al. 2014, Frantz et al. 2012). Despite the known association between UA concentration and the clinical markers of $\mathrm{HF}$, evidence supporting the independent association between UA and NT-proBNP concentrations were rather weak. Li et al. (2016) found an association between UA and the clinical markers of HF severity, such as NYHA; however, no association with NT-proBNP was observed. One cross sectional study found a strong positive association between NT-proBNP and UA (Zhang et al. 2009). However, multivariate analysis was not used, and their results were not cleared of the effects of potential confounding factors. Both UA and NT-proBNP concentrations might be influenced by CCR, age, gender, losartan therapy and BMI, which could lead to false positivity. To minimize the chance of false positivity, we conducted partial Spearman correlation, adjusting UA based on previously mentioned control variables such CCR, age, gender, losartan therapy and BMI. According to this analysis, UA independently correlated with NT-proBNP; however, the association was rather weak and much weaker than that observed by Zhang et al. (2009). This indicates the substantial effect of confounding factors. Impaired CCR as a marker of glomerular function represents possibly the strongest confounding factor. HF impairs glomerular function, and $\mathrm{HF}$ severity is independently negatively associated with CCR (Lu et al. 2014). Since UA is eliminated by the kidneys, UA rises with CCR decline (Johnson et al. 2013). Another possible confounding factor is the age (Hill et al. 2014). Our results, however, for the first time, indicates that a significant part of the association between UA and NT-proBNP concentrations cannot be attributed to possible confounding factors and therefore, might be explained by a harmful pathophysiological interaction between UA and the failing heart. Our findings support the theory of the connection between hyperuricemia and progression of HF; however, the pathophysiological causality remains to be elucidated. There is some evidence that the treatment of hyperuricemia with allopurinol or oxypurinol in $\mathrm{HF}$ patients might improve the heart failure prognosis (Thanassoulis et al. 2010, Hare et al. 2008). This, similar to our results, supports the theory that high UA concentration may, indeed, play a significant role in the pathogenesis of HF progression and could be considered as an independent risk factor instead of just as a marker of poor prognosis in HF. However, more studies are needed to conclusively determine whether UA could be a new potential therapeutic target in the treatment of $\mathrm{HF}$ in common clinical practice.

UA concentration and left ventricle systolic

function: In our study, the UA concentration adjusted for gender, CCR, age, losartan therapy and BMI in the group of patients with HFrEF was significantly higher than in groups with HFmrEF and HFpEF. Difference in the concentration of UA between groups of patients with HFpEF and HFmrEF was not statistically significant. According to our results, only HFrEF (LVEF lower than $40 \%$ ) was associated with increased UA concentration. According to the study by Pinelli et al. (2007) UA concentration negatively correlates with LVEF; however, they did not compare the levels of UA according to systolic dysfunction stages by LVEF impairment (Pinelli et al. 2007).

Pathogenesis of $\mathrm{UA}$ and $\mathrm{HF}$ association: Proposed pathogenesis of heart failure is rather complex (Simko et al. 2014, Simko and Adamcova 2018, Cundrle et al. 2018). There are various hypotheses that explain the pathophysiologic background of the increased UA concentration in the development and progression of HF. Production of UA is catalyzed by xanthine oxidase (XO) in a reaction that also leads to the creation of reactive oxygen species (ROS) (Sougstad et al. 1996). It is known, that ROS plays a significant role in the pathogenesis of failing myocardium (Wang et al. 2018, Liu et al. 2018). High UA concentration may reflect increased XO activity and excessive ROS formation, which results in a higher burden of free radicals (McCord et al. 1985). XO upregulation has been identified in the myocardium of HF patients (Cappola et al. 2001). In the pathogenesis of $\mathrm{HF}, \mathrm{XO}$ activity participates in a mechanoenergetic uncoupling of the myocardium, which means a decrease in contractility despite a normal or increased energetic consumption. Mechanoenergetic uncoupling might be mediated by the negative effect of ROS, produced by XO, on creatine kinase activity in the myocytes (Hare et al. 2003). These potential relations between UA, oxygen stress and deterioration of HF are supported by the finding that intracoronary infusion of allopurinol blocking the XO activity accelerates the restoration of an impaired myocardial energy metabolism (Cappola et al. 2001). Blocking of XO activity might be responsible for beneficial effects of allopurinol. Recently published results of FREED study showed that another UA lowering compound, XO inhibitor febuxostat is even 
capable to decrease the incidence of heart failure requiring hospitalization, nonfatal cardiovascular events and the rate of progression of chronic renal disease (Kojima et al. 2019). It further supports the hypothesis that excessive $\mathrm{XO}$ activity might stand in the pathophysiological background of the UA and HF relation. High UA concentration is a negative prognostic marker not just for $\mathrm{HF}$ progression, but it seems to be associated also with other cardiovascular pathologies and even with increased cardiovascular mortality. Therefore, the negative effects of UA on the severity of HF might also be mediated by the worsening of the coronary heart disease or hypertension as a result of high level of UA (Timóteo et al. 2013, Qin et al. 2016). On the other hand, HF itself may in the sequence of events result in the secondary enhancement of UA concentration, which is in normal conditions excreted by the kidneys. However, severe heart failure may result in the impairment of kidney function and deterioration of excretion of the excessive amount of UA, supporting, thus, the vicious circle of the UA accumulation and its heart damaging effect. Indeed, a negative correlation between CCR and NT-proBNP was found by previous studies (Lu et al. 2014, Johnson et al. 2013) and our study. This mismatch could be further supported by the hypoperfusion of peripheral tissues in HF, resulting in accelerated breakdown of ATP to adenosine and hypoxanthine with its final conversion to UA. Moreover, the peripheral tissue ischemia may result to the accumulation of lactic acid competing with UA excretion in kidneys (Hare et al. 2003). It should also be considered that UA itself acts as a compound with potent antioxidative properties (Fabbrine et al. 2014). Moreover, the association of UA concentration and cardiovascular mortality and all-cause mortality is rather U-shaped than linear, possibly because of malnutrition associated with very low UA concentration (Tseng et al. 2018). Thus, the pathophysiologic background of UA and HF association seems to be complex and needs further study. Data of this work, the results of FREED study and other previously mentioned studies support the idea that there is a direct pathological link between UA metabolism and heart failure and suggest that the association of UA concentration and HF development and progression might be causal and not only determined by possible confounding factors. However, it remains unclear, whether this link is mediated trough the excessive $\mathrm{XO}$ activity and ROS formation, direct toxic effect of UA or some other mechanisms.

\section{Conclusions}

In a Slovak population of patients suffering from heart failure, uric acid concentration correlated with NT-proBNP concentration independently of age, BMI, and creatinine clearance in both sexes. Uric acid concentration was also positively associated with heart failure severity indicated by NYHA class and degree of systolic dysfunction and with diastolic dysfunction. Hyperuricemia was associated with symptomatic heart failure and impairment of systolic function and diastolic functions and left ventricular hypertrophy. Our results support the role of uric acid as an important biochemical marker of heart failure severity and prognosis.

\section{Conflict of Interest}

There is no conflict of interest.

\section{References}

BENJAMIN EJ, BLAHA MJ, CHIUVE SE, CUSHMAN M, DAS SR, DEO R, FERRANTI SD, FLOID J, FORNAGE M, GILLESPIE C, ISASI CR, JIMÉNEZ MC, JORDAN LC, JUDD SE, LACKLAND D, LICHTMAN JH, LISABETH L, LIU S, LONGENECKER CL, MACKEY RH, MATSUSHITA K, MOZAFFARIAN D, MUSSOLINO ME, NASIR K, NEUMAR RW, PALANIPPAN L, PANDEY DK, THIAGARAJAN RR, REEVES MJ, RITCHEY M, RODRIGUEZ CJ, ROTH GA, ROSAMOND WD, SASSON C, TOWFIGHI A, TSAO CW, TURNER MB, VIRANI SS, VOEKS JH, WILLEY JZ, WILKINS JT, WU JH, ALGER HM, WONG SS, MUNTHER P: American Heart Association Statistics Committee and Stroke Statistics Subcommittee. Heart disease and stroke statistics-2017 update: a report from the American Heart Association. Circulation 135: e146-e603, 2017.

BUI AL, HORWITCH TB, FONAROW GC: Epidemiology and risk profile of heart failure. Nat Rev Cardiol 8: 30-41, 2011. 
CAPPOLA TP, KASS DA, NELSON GS, BERGER RD, ROSAS GO, KOBEISSI ZA, MARBÁN E, HARE JM: Allopurinol improves myocardial efficiency in patients with idiopathic dilated cardiomyopathy. Circulation 104: 2407-2411, 2001.

CIBICKOVA L, LANGOVA K, VAVERKOVA H, KUBICKOVA V, KARASEK D: Correlation of uric acid levels and parameters of metabolic syndrome. Physiol Res 66: 481-487, 2017.

CUNDRLE I JR, SUK P, SRAMEK V, LACINOVA Z, HALUZIK M: Circadian leptin concentration changes in critically ill heart failure patients. Physiol Res 67: 505-508, 2018.

FANG J, ALDERMAN MH: Serum uric acid and cardiovascular mortality. The NHANES I epidemiologic follow-up study, 1971-1992. JAMA 283: 2404-2410, 2000.

FABBRINI E, SERAFINI M, COLIC BARIC I, HANZEN SL, KLEIN S: Effect of plasma uric acid on antioxidant capacity, oxidative stress, and insulin sensitivity in obese subjects. Diabetes 63: 976-981, 2014.

FRANTZ RP, MCDEVITT S, WALKER S: Baseline NT-proBNP correlates with change in 6-minute walk distance in patients with pulmonary arterial hypertension in the pivotal inhaled treprostinil study TRIUMPH-1. J Heart Lung Transplant 31: 811-816, 2012.

GETLER MM, GARN SM, LEVINE SA: Serum uric acid in relation to age and physique in health and coronary heart disease. Ann Intern Med 34: 1421-1431, 1951.

HARE JM, JOHNSON RJ: Uric acid predicts clinical outcomes in heart failure: insights regarding the role of xanthine oxidase and uric acid in disease pathophysiology. Circulation 107: 1951-1953, 2003.

HAREJM, MANGAL B, BROWN J, FISCHER C JR, FREUDENBERGER R, COLUCCI WS, MANN DL, LIU P, GIVERTZ MM, SCHWARZ RP, OPT-CHF Investigators: impact of oxypurinol in patients with symptomatic heart failure. Results of the OPT-CHF study. J Am Coll Cardiol 51: 2301-2309, 2008.

HILL SA, BOOTH RA, SANTAGUIDA PL, DON-WAUCHOPE A, BROWN JA, OREMUS M, ALI U, BUSTAMAM A, SOHEL N, MCKELVIE R, BAILON C, RAINA P: Use of BNP and NT-proBNP for the diagnosis of heart failure in the emergency department: a systematic review of the evidence. Heart Fail Rev 19: 421-438, 2014.

HUANG H, HUANG B, LI Y, HUANG Y, LI J, YAO H, JING X, CHEN J, WANG J: Uric acid and risk of heart failure: a systematic review and meta-analysis. Eur J Heart Fail 16: 15-24, 2014.

JOHNSON RJ, NAKAGAWA T, JALAL D, SANCHEZ-LOZADA LG, KANG DH, RITZ E: Uric acid and chronic kidney disease: which is chasing which? Nephrol Dial Transplant 28: 2221-2228, 2013.

KIM HN, JANUZZI JL JR: Natriuretic peptide testing in heart failure. Circulation 123: 2015-2019, 2011.

KOJIMA S, MATSUI K, HIRAMITSU S, HISATOME I, WAKI M, UCHIYAMA K, YOKOTA N, TOKUTAKE E, WAKASA Y, JINNOUCHI H, KAKUDA H, HAYASHI T, KAWAI N, MORI H, SUGAWARA M, OHYA Y, KIMURA K, SAITO Y, OGAWA H; Febuxostat for Cerebral and CaRdiorenovascular Events PrEvEntion StuDy (FREED) investigators: Febuxostat for Cerebral and CaRdiorenovascular Events PrEvEntion StuDy. Eur Heart J 40: 1778-1786, 2019.

LACKO S, MLCEK M, HALA P, POPKOVA M, JANAK D, HRACHOVINA M, KUDLICKA J, HRACHOVINA V, OSTANDAL P, KITTNAR O: Severe acute heart failure - experimental model with very low mortality. Physiol Res 67: 555-562, 2018.

LI L, SUN M, ZHANG Y: Serum uric acid, NT-ProBNP and hs-CRP as biomarkers in chronic heart failure. Int J Clin Exp Med 9: 18324-18331, 2016.

LIU JJ, LU Y, PING NN, LI X, LIN YX, LI CF: Apocynin ameliorates pressure overload-induced cardiac remodeling by inhibiting oxidative stress and apoptosis. Physiol Res 66: 741-752, 2017.

LU Y, WANG B, WANG Z, QIAN X, ZHENG W, WEI M: Relationship between CCR and NT-proBNP in Chinese HF Patients, and Their Correlations with Severity of HF. BioMed Res Int 2014: 106252, 2014.

MCCORD JM: Oxygen-derived free radicals in postischemic tissue injury. $N$ Eng J Med 12: 159-163, 1985.

PINELLI M, BINDI M, FILARDO FP, MORONLI F, CASTIGLIONI M: Serum uric acid levels correlate with left ventricular ejection fraction and systolic pulmonary artery pressure in patients with heart failure. (In Italian) Recenti Prog Med 98: 619-623, 2007. 
PONIKOWSKI P, VOORS AA, ANKER SD, BUENO H, CLELAND JG, COATS AJ, FALK V, GONZÁLEZJUANATEY JR, HARJOLA VP, JANKOWSKA EA, JESSUP M, LINDE C, NIHOYANNOPOULOS P, PARISSIS JT, PIESKE B, RILEY JP, ROSANDO GM, RUILOPE LM, RUSCHITZKA F, RUTTEN FH, VAN DER MEER P: Authors/Task Force Members; Document Reviewers. 2016 ESC Guidelines for the diagnosis and treatment of acute and chronic heart failure: The Task Force for the diagnosis and treatment of acute and chronic heart failure of the European Society of Cardiology (ESC). Developed with the special contribution of the Heart Failure Association (HFA) of the ESC. Eur J Heart Fail 18: 891-975, 2016.

QIN T, ZHOU X, WANG J, WU X, LI Y, WANG L, HUANG H, LI J: Hyperuricemia and the prognosis of hypertensive patients: a systematic review and meta-analysis. J Clin Hypertens 18: 1268-1278, 2016.

RUILOPE LM, DUKAT A, BOHM M, LACOURCIERE Y, GONG J, LEFKOWITZ MP: Blood-pressure reduction with LCZ696, a novel dual-acting inhibitor of the angiotensin II receptor and neprilysin: a randomised, doubleblind, placebo-controlled, active comparator study. Lancet 375: 1255-1266, 2010.

SAUGSTAD OD: Role of xanthine oxidase and its inhibitor in hypoxia: reoxygenation injury. Pediatrics 98: 103-107, 1996.

SIMKO F, SIMKO J: Heart failure and angiotensin converting enzyme inhibition: problems and perspectives. Physiol Res 48: 1-8, 1999.

SIMKO F, SIMKO J, FABRYOVA M: ACE-inhibition and angiotensin II receptor blockers in chronic heart failure: pathophysiological consideration of the unresolved battle. Cardiovasc Drugs Ther 17: 287-290, 2003.

SIMKO F, PECHANOVA O: Remodelling of the heart and vessels in experimental hypertension: advances in protection. J Hypertens 28 (Suppl 1): S1-S6, 2010.

SIMKO F, BEDNAROVA K, KRAJCIROVICOVA K, HRENAK J, CELEC P, KAMODYOVA N, GAJDOSECHOVA L, ZORAD S, ADAMCOVA M: Melatonin reduces cardiac remodeling and improves survival in rats with isoproterenol-induced heart failure. J Pineal Res 57: 177-184, 2014.

SIMKO F, ADAMCOVA M: What prevents cardioprotective drugs from reaching the market? Expert Rev Clin Pharmacol 11: 463-465, 2018.

SIMKO F, BAKA T, KRAJCIROVICOVA K, REPOVA K, AZIRIOVA S, ZORAD S, POGLITSCH M, ADAMCOVA M, REITER RJ, PAULIS L: Effect of melatonin on the renin-angiotensin-aldosterone system in L-NAME-induced hypertension. Molecules 23: E265, 2018a.

SIMKO F, BAKA T, POGLITSCH M, REPOVA K, AZIRIOVA S, KRAJCIROVICOVA K, ZORAD S, ADAMCOVA M, PAULIS L: Effect of ivabradine on a hypertensive heart and the renin-angiotensinaldosterone system in L-NAME-induced hypertension. Int J Mol Sci 19: pii: E3017, 2018 b.

TAMARIZ L, HARZAND A, PALACIO A, VERMA S, JONES J, HARE J: Uric acid as a predictor of all-cause mortality in heart failure: a meta-analysis. Congest Heart Fail 17: 25-30, 2011.

THANASSOULIS G, BROPHY JM, RICHARD H, PILOTE L: Gout, allopurinol use, and heart failure outcomes. Arch Intern Med 170: 1358-1364, 2010.

TIMOTEO AT, LOUSINHA A, LABANDEIRO J, MIRANDA F, PAPOILA AL, OLIVEIRA JA, FERREIRA ML, FERREIRE LC: Serum uric acid: a forgotten prognostic marker in acute coronary syndromes? Eur Heart $J$ Acute Cardiovasc Care 2: 44-52, 2013.

TSENG WC, CHEN YT, OU SM, SHIH CJ, TAMG DC; Taiwan Geriatric Kidney Disease (TGKD) Research Group: U-shaped association between serum uric acid levels with cardiovascular and all-cause mortality in the elderly: the role of malnourishment. $J$ Am Heart Assoc 7: e007523, 2018.

WANG JP, CHI RF, LIU J, DENG YZ, HAN XB, QIN FZ, LI B: The role of endogenous reactive oxygen species in cardiac myocyte autophagy. Physiol Res 67: 31-40, 2018.

WILLIAMS SG, NG LL, O'BRIEN RJ, TAYLOR S, WRIGHT DJ, LI YF, LI YF, TAN LB: Complementary roles of simple variables, NYHA and N-BNP, in indicating aerobic capacity and severity of heart failure. Int $J$ Cardiol 102: 279-286, 2005.

ZHANG YH, LÜ R, ZHAO XY, KANG LM, YANG YJ, ZHANG J: Association of serum uric acid, plasma NT-proBNP, Hs-C reactive protein and invasive hemodynamic parameters in patients with heart failure. (Article in Chinese) Zhonghua Xin Xue Guan Bing Za Zhi 37: 126-129, 2009. 